\title{
Response of Hybrid Rice (Oryza sativa L.) to Different Dates of Transplanting, Spacing and Number of Seedlings/Hill
}

\author{
Shivam Dubey ${ }^{1}$, Rajesh Singh ${ }^{1 *}$, A.C. Singh ${ }^{2}$ and S.P. Vishwakarma ${ }^{2}$ \\ ${ }^{1}$ Department of Agronomy, SHUATS, Allahabad Department of Agronomy, SHIATS, \\ Allahabad-211007 (U.P), India \\ ${ }^{2}$ Kulbhaskar Ashram Degree College, India \\ *Corresponding author:
}

\begin{tabular}{|c|c|}
\hline & A B S T R A C T \\
\hline Keywords & \multirow{4}{*}{$\begin{array}{l}\text { A field experiment was conducted during kharif season } 2011 \text { at Crop Research } \\
\text { Farm, Department of Agronomy, SHIATS, Allahabad. The experiment } \\
\text { consisted } 18 \text { treatments, laid out in RBD and replicated thrice. The treatments } \\
\text { included three different dates of transplanting }\left(15^{\text {th }} \mathrm{July}, 27^{\text {th }} \mathrm{July} \text { and } 9^{\text {th }}\right. \\
\text { August), three spacing's }(20 \mathrm{~cm} \times 10 \mathrm{~cm}, 20 \mathrm{~cm} \times 15 \mathrm{~cm} \text { and } 20 \mathrm{~cm} \times 20 \mathrm{~cm}) \text { and } \\
\text { two seedling rates }\left(1 \text { seedling hill }{ }^{-1} \text { and } 2 \text { seedling hill } l^{-1}\right) \text { in hybrid rice. Out of } \\
\text { them treatment } \mathrm{T}_{6}\left(15^{\text {th }} \text { July transplanting, } 2 \text { seedling } / \text { hill and } 20 \mathrm{~cm} \times 20 \mathrm{~cm}\right. \\
\text { spacing) recorded the maximum value for plant height, no. of tillers } / \text { hill, no. of } \\
\text { effective tillers } / \text { hill, plant dry weight and RGR. The yield attributes namely no. } \\
\text { of grain panicle }{ }^{-1} \text {, length of panicle (cm), test weight, straw yield and } \\
\text { maximum grain yield were also obtained in the aforesaid treatment. }\end{array}$} \\
\hline $\begin{array}{l}\text { Hybrid rice, Dates } \\
\text { of transplanting, } \\
\text { Plant geometry, } \\
\text { Number of } \\
\text { seedlings / hill. }\end{array}$ & \\
\hline Article Info & \\
\hline $\begin{array}{l}\text { Accepted: } \\
\text { 23 May } 2017 \\
\text { Available Online: } \\
\text { 10 June } 2017\end{array}$ & \\
\hline
\end{tabular}

\section{Introduction}

Rice (Oryza sativa L.) is the world's most important food crops of Asian origin. It is an important food grain crop grown extensively in tropical and subtropical region of the world. About 90 per cent of all rice grown in the world is produced and consumed in the Asian region. Rice production always plays a key role in the global food situation as well as in commercial industrial preparations.

Rice is staple food of more than $60 \%$ of Indian population. It accounts for about $43 \%$ of total food grain production and $46 \%$ of total cereal production in the country. In order to meet the domestic demand of the increasing population the present day production of 99 million tones of milled rice has to be increased to130 million tones by the year 2025. In Uttar Pradesh the area under rice is $5.63 \mathrm{~m}$ ha, production of $11.94 \mathrm{mt}$ and productivity of $21.20 \mathrm{Q} / \mathrm{ha}$.

Rice is an excellent source of carbohydrate and protein of regular human diet. It is a staple food of more than two thirds of world population. The slogan "Rice is life" is most appropriate for India as this crop plays a vital role in our national food security and is a means of livelihood for millions of rural people in India. 
Transplanting rice in the optimum period of time is critical to achieve high grain yield. However, optimum rice planting dates are regional and vary with location and genotypes.

Rice plants require a particular temperature for its phonological affair such as panicle initiation; flowering, panicle exertions from flag leaf sheath and maturity and these are very much influenced by the planting dates during India season. Rice seeded before the window of optimum dates usually has slow germination and emergence, poor stand establishment and increased damages from soil borne seedling diseases.

\section{Materials and Methods}

A field experiment was conducted during Kharif season 2011 at Crop Research Farm, Department of Agronomy, Allahabad School of Agriculture, SHIATS, Allahabad. The soil was sandy loam in texture having $7.93 \mathrm{pH}$, $0.34 \%$ organic carbon, $85.45 \mathrm{~kg} / \mathrm{ha}$ total $\mathrm{N}$ and available $\mathrm{P}_{2} \mathrm{O}_{5}$ and $\mathrm{K}_{2} \mathrm{O}$ of 18.25 and $150.34 \mathrm{~kg} \mathrm{ha}^{-1}$, respectively. The experiment consisted different dates of transplanting $\left(15^{\text {th }}\right.$ July, $27^{\text {th }}$ July and $9^{\text {th }}$ August), spacing's $(20 \mathrm{~cm} \times 10 \mathrm{~cm}, 20 \mathrm{~cm} \times 15 \mathrm{~cm}$ and $20 \mathrm{~cm} \times$ $20 \mathrm{~cm})$ and seedling rates $\left(1\right.$ seedling hill $^{-1}$ and 2 seedling hill $^{-1}$ ) were tested in three replicated Randomized Block Design and replicated thrice.

Twenty one days old seedlings of hybrid rice 'Arize 6444' were transplanted on each date using uniform dose of $120 \mathrm{~kg} \mathrm{~N}, 80 \mathrm{~kg} \mathrm{P}_{2} \mathrm{O}_{5}$ and $60 \mathrm{~kg} \mathrm{~K}_{2} \mathrm{O}$ per ha ${ }^{-1}$ along with $30 \mathrm{~kg}$ zinc sulphate $\mathrm{ha}^{-1}$. The half dose of $\mathrm{N}$ and entire dose of $\mathrm{P}, \mathrm{K}$ and $\mathrm{Zn}$ were applied at the time of puddling and rest of $\mathrm{N}$ was given in 2 equal splits, at tillering and panicle initiation stages. Arize 6444 variety was used as planting materials in this study.

\section{Results and Discussion}

\section{Plant height (cm)}

The observations on the plant height of rice recorded at different interval were statistically analyzed and are being presented in table 1 . A perusal of the table clearly shows that at 60 DAT the highest plant height $77.41 \mathrm{~cm}$ was recorded in treatment $\mathrm{T}_{2}$ (15 July + spacing of $20 \times 10 \mathrm{~cm}+2$ Seedling hill $\left.{ }^{-1}\right)$ although the difference amongst the treatments were nonsignificant. At 90 DAT significantly higher plant height $92.24 \mathrm{~cm}$ was recorded in treatment $\mathrm{T}_{6}(15$ July $+20 \times 20 \mathrm{~cm}+2$ Seedling hill $\left.{ }^{-1}\right)$.

More duration and higher heat accumulation might have favored most of the growth parameters in July $17^{\text {th }}$ planting as compared to $24^{\text {th }}$ and $31^{\text {th }}$ July planting. These results confirm the findings of (Gautam et al., 2003) and Nayak et al., (2003).

\section{Plant dry weight (g)}

The observations on response of hybrid rice on the plant dry weight at different intervals was statistically analyzed and is being presented in table 1. A perusal of the table clearly shows that at 60 DAT the highest dry matter $(3.70 \mathrm{~g})$ was recorded in treatment $\mathrm{T}_{1}$ $\left(15\right.$ July $+20 \times 10 \mathrm{~cm}+1$ Seedling hill $\left.{ }^{-1}\right)$ which was found to be non significant and at 90 DAT significantly higher dry matter accumulation (17.33g) was recorded in treatment $\mathrm{T}_{6}(15 \mathrm{July}+20 \times 20 \mathrm{~cm}+2$ Seedling hill $\left.{ }^{-1}\right)$.

$16^{\text {th }}$ July of transplanting of rice found significantly higher dry weight of plant. Dry matter accumulation was low in late transplanting, this may due to prevalence of low temperature coupled with less humidity at the reproductive stage or at flag leaf stage might have reduced the yield as compared to 
earlier planting. These results confirm the findings of Yadav and Tripathi (2008).

\section{No. of tillers hill ${ }^{-1}$}

The observations on response of hybrid rice to the total number of tillers/hill recorded at different intervals were statistically analyzed and are being presented in table 2 . A critical review of the table clearly shows that at 90 DAT the highest no. of tillers per hill was recorded in treatment $\mathrm{T}_{6}(15 \mathrm{July}+20 \times 20 \mathrm{~cm}$ +2 Seedling hill ${ }^{-1}$ ) which was found to be significant. At 60 DAT the highest no. of tillers per hill was observed in treatment $\mathrm{T}_{15}$ $\left(09\right.$ August $+20 \times 15 \mathrm{~cm}+1$ Seedling hill $\left.{ }^{-1}\right)$ which was non-significant.

July $14^{\text {th }}$ transplanting crop was observed more duration and higher heat accumulation which might have favored most of the growth parameters. In the early transplanting improves the canopy's photosynthesis and increase tillers (Kumar et al., 1998).

\section{Crop growth rate (CGR)}

A critical review of the table clearly shows that at 30 DAT the highest crop growth rate $\left(2.50 \mathrm{~g} / \mathrm{m}^{2} /\right.$ day $)$ was recorded in $\mathrm{T}_{13}(09$ August $+20 \times 10 \mathrm{~cm}+1$ Seedling hill $\left.{ }^{-1}\right)$. At 60 DAT highest CGR (4.50 g/m²/day) was observed in treatment $T_{1}(15$ July $+20 \times 10 \mathrm{~cm}$ +1 Seedling hill $\left.{ }^{-1}\right)$. Although the difference among the treatments was non-significant both at 30 and 60 DAT. At 90 DAT significantly higher crop growth rate (18.57 $\mathrm{g} / \mathrm{m}^{2} /$ day) was recorded in treatment $\mathrm{T}_{2}(15$ July $+20 \times 10 \mathrm{~cm}+2$ Seedling hill ${ }^{-1}$ ), than all the other treatments.

\section{Relative growth rate (RGR)}

A perusal of the table clearly shows that at 30-60 DAT the highest relative growth rate (0.0537 g/g/day) was recorded under treatment $\mathrm{T}_{5}(15 \mathrm{July}+20 \times 20 \mathrm{~cm}+1$ Seedling hill $\left.^{-1}\right)$. At 60-90 DAT the higher RGR (0.0578 $\mathrm{g} / \mathrm{g} /$ day) was recorded in treatment $\mathrm{T}_{6}(15$ July $+20 \times 20 \mathrm{~cm}+2$ Seedling hill ${ }^{-1}$ ). However, the difference was non significant among at the different treatments at both the intervals.

\section{Number of effective tillers/hill}

The observations on response of hybrid rice on the number of panicles were statistically analyzed and are being presented in table 2 . A critical review of the table clearly shows that the significantly higher number of effective tillers/hill (33.27) was recorded under treatment $\mathrm{T}_{6}(15 \mathrm{July}+20 \times 20 \mathrm{~cm}+2$ Seedling hill $^{-1}$ ).

Effective tillers production was low in late transplanting. This may due to prevalence of low temperature coupled with less humidity at the time of reproductive stages (Kumar et al., 2006).

Transplanting of rice at a wider spacing of $20 \times 20 \mathrm{~cm}^{2}$ produces a significantly higher number of effective tillers/hill as compared to closer spacing of $20 \times 10 \mathrm{~cm}^{2}$ and $20 \times 15 \mathrm{~cm}^{2}$ (Gautam et al., 2008).

\section{Length of panicle $(\mathrm{cm})$}

The observations on response of hybrid rice on the length of panicle were statistically analyzed and are being presented in table 2 . A perusal of the table clearly shows that the significantly higher length of panicle (27.33) was recorded under treatment $\mathrm{T}_{6}$ (15 July $+20 \times 20 \mathrm{~cm}+2$ Seedling hill ${ }^{-1}$ ).

Yaday and Tripathi (2008) reported that maximum panicle length $(26.63 \mathrm{~cm})$ was found to be at the transplanting date $15^{\text {th }}$ July with $20 \times 20 \mathrm{~cm}$ plant spacing and 2 seedlings/hill as compare to other transplanting dates, $30^{\text {th }}$ July and $14^{\text {th }}$ August. 


\section{Number of grains/panicle}

The observations on response of hybrid rice on the number of grains per panicle were statistically analyzed and are being presented in table 2. A perusal of the table clearly shows that the significantly higher number of grains/panicle (202.33) was recorded under treatment $\mathrm{T}_{6}(15 \mathrm{July}+20 \times 20 \mathrm{~cm}+2$ Seedling hill $^{-1}$ ). Number of grains/panicle, was significantly higher in $15^{\text {th }}$ July planted crop than the $30^{\text {th }}$ July and $14^{\text {th }}$ August planted crop which might be due to availability of more time for better development of plant parts which may result in the better development of yield attributing characters.

Table.1a Effect of different transplanting dates, spacing and No. of seedlings/hill on growth attributes of hybrid rice at different Intervals

\begin{tabular}{|c|c|c|c|c|c|}
\hline \multirow[t]{2}{*}{+2} & \multirow[t]{2}{*}{ Treatments combination } & \multicolumn{2}{|c|}{ Plant height (cm) } & \multicolumn{2}{|c|}{ Dry weight (g) } \\
\hline & & 60 DAT & 90 DAT & 60 DAT & 90 DAT \\
\hline $\mathrm{T} 1$ & $15^{\text {th }}$ July, sowing of 1 seedling/ hill at $20 \mathrm{~cm} \times 10 \mathrm{~cm}$ spacing & 66.85 & 71.89 & 3.70 & 13.88 \\
\hline $\mathrm{T} 2$ & $15^{\text {th }}$ July, sowing of 2 seedling/ hill at $20 \mathrm{~cm} \times 10 \mathrm{~cm}$ spacing & 77.41 & 71.97 & 2.86 & 14.00 \\
\hline $\mathrm{T} 3$ & $15^{\text {th }}$ July, sowing of 1 seedling/ hill at $20 \mathrm{~cm} \times 15 \mathrm{~cm}$ spacing & 75.07 & 78.69 & 3.17 & 15.07 \\
\hline $\mathrm{T} 4$ & $15^{\text {th }}$ July, sowing of 2 seedling/ hill at $20 \mathrm{~cm} \times 15 \mathrm{~cm}$ spacing & 76.22 & 80.09 & 3.42 & 15.33 \\
\hline T5 & $15^{\text {th }}$ July, sowing of 1 seedling/ hill at $20 \mathrm{~cm} \times 20 \mathrm{~cm}$ spacing & 71.88 & 90.22 & 3.44 & 16.67 \\
\hline T6 & $15^{\text {th }}$ July, sowing of 2 seedling/ hill at $20 \mathrm{~cm} \times 20 \mathrm{~cm}$ spacing & 74.42 & 92.24 & 3.12 & 17.33 \\
\hline $\mathrm{T} 7$ & $27^{\text {th }}$ July, sowing of 1 seedling/ hill at $20 \mathrm{~cm} \times 10 \mathrm{~cm}$ spacing & 72.15 & 70.55 & 2.67 & 13.44 \\
\hline $\mathrm{T} 8$ & $27^{\text {th }}$ July, sowing of 2 seedling/ hill at $20 \mathrm{~cm} \times 10 \mathrm{~cm}$ spacing & 69.13 & 70.61 & 3.47 & 13.67 \\
\hline T9 & $27^{\text {th }}$ July, sowing of 1 seedling/ hill at $20 \mathrm{~cm} \times 15 \mathrm{~cm}$ spacing & 68.87 & 73.28 & 3.35 & 14.67 \\
\hline $\mathrm{T} 10$ & $27^{\text {th }}$ July, sowing of 2 seedling/ hill at $20 \mathrm{~cm} \times 15 \mathrm{~cm}$ spacing & 72.12 & 75.01 & 3.19 & 14.88 \\
\hline $\mathrm{T} 11$ & $27^{\text {th }}$ July, sowing of 1 seedling/ hill at $20 \mathrm{~cm} \times 20 \mathrm{~cm}$ spacing & 66.60 & 89.83 & 3.23 & 16.17 \\
\hline $\mathrm{T} 12$ & $27^{\text {th }}$ July, sowing of 2 seedling/ hill at $20 \mathrm{~cm} \times 20 \mathrm{~cm}$ spacing & 62.99 & 89.83 & 3.13 & 16.44 \\
\hline $\mathrm{T} 13$ & $9^{\text {th }}$ Aug., sowing of 1 seedling/ hill at $20 \mathrm{~cm} \times 10 \mathrm{~cm}$ spacing & 60.41 & 68.67 & 3.33 & 11.88 \\
\hline T14 & $9^{\text {th }}$ Aug., sowing of 2 seedling/ hill at $20 \mathrm{~cm} \times 10 \mathrm{~cm}$ spacing & 58.22 & 69.77 & 3.03 & 12.77 \\
\hline $\mathrm{T} 15$ & $9^{\text {th }}$ Aug., sowing of 1 seedling/ hill at $20 \mathrm{~cm} \times 15 \mathrm{~cm}$ spacing & 65.49 & 72.15 & 3.06 & 14.17 \\
\hline $\mathrm{T} 16$ & $9^{\text {th }}$ Aug., sowing of 2 seedling/ hill at $20 \mathrm{~cm} \times 15 \mathrm{~cm}$ spacing & 57.84 & 72.78 & 3.41 & 14.33 \\
\hline T17 & $9^{\text {th }}$ Aug., sowing of 1 seedling/ hill at $20 \mathrm{~cm} \times 20 \mathrm{~cm}$ spacing & 63.40 & 84.86 & 3.24 & 15.55 \\
\hline \multirow[t]{3}{*}{$\mathrm{T} 18$} & $9^{\text {th }}$ Aug., sowing of 2 seedling/ hill at $20 \mathrm{~cm} \times 20 \mathrm{~cm}$ spacing & 60.89 & 85.64 & 3.26 & 15.77 \\
\hline & F-test & NS & S & NS & S \\
\hline & C.D. $(P=0.05)$ & - & 0.09 & - & 0.09 \\
\hline
\end{tabular}


Table.1b Effect of different transplanting dates, spacing and No. of seedlings/hill on the growth attributes of hybrid rice

\begin{tabular}{|c|c|c|c|c|c|}
\hline & Treatments combination & & & & \\
\hline & & $\begin{array}{l}\text { 30-60 } \\
\text { DAT }\end{array}$ & $\begin{array}{l}\text { 60-90 } \\
\text { DAT }\end{array}$ & $\begin{array}{l}\text { 30-60 } \\
\text { DAT }\end{array}$ & $\begin{array}{l}\text { 60-90 } \\
\text { DAT }\end{array}$ \\
\hline $\mathrm{T}_{1}$ & $\begin{array}{l}15^{\text {th }} \mathrm{July} \text {, sowing of } 1 \text { seedling/ hill at } \\
20 \mathrm{~cm} \times 10 \mathrm{~cm} \text { spacing }\end{array}$ & 4.50 & 16.96 & 0.0504 & 0.0445 \\
\hline $\mathrm{T}_{2}$ & $\begin{array}{l}15^{\text {th }} \text { July, sowing of } 2 \text { seedling/ hill at } \\
20 \mathrm{~cm} \times 10 \mathrm{~cm} \text { spacing }\end{array}$ & 2.18 & 18.57 & 0.0210 & 0.0533 \\
\hline $\mathrm{T}_{3}$ & $\begin{array}{l}15^{\text {th }} \text { July, sowing of } 1 \text { seedling/ hill at } \\
20 \mathrm{~cm} \times 15 \mathrm{~cm} \text { spacing }\end{array}$ & 2.63 & 13.09 & 0.0476 & 0.0520 \\
\hline $\mathrm{T}_{4}$ & $\begin{array}{l}15^{\text {th }} \text { July, sowing of } 2 \text { seedling/ hill at } \\
20 \mathrm{~cm} \times 15 \mathrm{~cm} \text { spacing }\end{array}$ & 2.23 & 13.10 & 0.0313 & 0.0502 \\
\hline $\mathrm{T}_{5}$ & $\begin{array}{l}15^{\text {th }} \text { July, sowing of } 1 \text { seedling/ hill at } \\
20 \mathrm{~cm} \times 20 \mathrm{~cm} \text { spacing }\end{array}$ & 2.19 & 11.02 & 0.0537 & 0.0529 \\
\hline $\mathrm{T}_{6}$ & $\begin{array}{l}15^{\text {th }} \text { July, sowing of } 2 \text { seedling/ hill at } \\
20 \mathrm{~cm} \times 20 \mathrm{~cm} \text { spacing }\end{array}$ & 1.46 & 17.95 & 0.0287 & 0.0578 \\
\hline $\mathrm{T}_{7}$ & $\begin{array}{l}27^{\text {th }} \text { July, sowing of } 1 \text { seedling/ hill at } \\
20 \mathrm{~cm} \times 10 \mathrm{~cm} \text { spacing }\end{array}$ & 2.97 & 11.84 & 0.0372 & 0.0539 \\
\hline $\mathrm{T}_{8}$ & $\begin{array}{l}27^{\text {th }} \text { July, sowing of } 2 \text { seedling/ hill at } \\
20 \mathrm{~cm} \times 10 \mathrm{~cm} \text { spacing }\end{array}$ & 3.83 & 16.99 & 0.0377 & 0.0457 \\
\hline $\mathrm{T}_{9}$ & $\begin{array}{l}27^{\text {th }} \text { July, sowing of } 1 \text { seedling/ hill at } \\
20 \mathrm{~cm} \times 15 \mathrm{~cm} \text { spacing }\end{array}$ & 2.77 & 12.46 & 0.0470 & 0.0497 \\
\hline $\mathrm{T}_{10}$ & $\begin{array}{l}27^{\text {th }} \text { July, sowing of } 2 \text { seedling/ hill at } \\
20 \mathrm{~cm} \times 15 \mathrm{~cm} \text { spacing }\end{array}$ & 2.21 & 12.86 & 0.0330 & 0.0519 \\
\hline $\mathrm{T}_{11}$ & $\begin{array}{l}27^{\text {th }} \text { July, sowing of } 1 \text { seedling/ hill at } \\
20 \mathrm{~cm} \times 20 \mathrm{~cm} \text { spacing }\end{array}$ & 1.26 & 10.78 & 0.0208 & 0.0539 \\
\hline $\mathrm{T}_{12}$ & $\begin{array}{l}27^{\text {th }} \text { July, sowing of } 2 \text { seedling/ hill at } \\
20 \mathrm{~cm} \times 20 \mathrm{~cm} \text { spacing }\end{array}$ & 1.62 & 11.09 & 0.0327 & 0.0557 \\
\hline$T_{13}$ & $\begin{array}{l}9^{\text {th }} \text { Aug., sowing of } 1 \text { seedling/ hill at } \\
20 \mathrm{~cm} \times 10 \mathrm{~cm} \text { spacing }\end{array}$ & 3.04 & 14.26 & 0.0293 & 0.0427 \\
\hline $\mathrm{T}_{14}$ & $\begin{array}{l}9^{\text {th }} \text { Aug., sowing of } 2 \text { seedling/ hill at } \\
20 \mathrm{~cm} \times 10 \mathrm{~cm} \text { spacing }\end{array}$ & 2.89 & 16.24 & 0.0286 & 0.0480 \\
\hline $\mathrm{T}_{15}$ & $\begin{array}{l}9^{\text {th }} \text { Aug., sowing of } 1 \text { seedling/ hill at } \\
20 \mathrm{~cm} \times 15 \mathrm{~cm} \text { spacing }\end{array}$ & 2.28 & 12.22 & 0.0414 & 0.0518 \\
\hline $\mathrm{T}_{16}$ & $\begin{array}{l}9^{\text {th }} \text { Aug., sowing of } 2 \text { seedling/ hill at } \\
20 \mathrm{~cm} \times 15 \mathrm{~cm} \text { spacing }\end{array}$ & 2.45 & 12.01 & 0.0350 & 0.0485 \\
\hline $\mathrm{T}_{17}$ & $\begin{array}{l}9^{\text {th }} \text { Aug., sowing of } 1 \text { seedling/ hill at } \\
20 \mathrm{~cm} \times 20 \mathrm{~cm} \text { spacing }\end{array}$ & 1.80 & 10.26 & 0.0392 & 0.0526 \\
\hline $\mathrm{T}_{18}$ & $\begin{array}{l}9^{\text {th }} \text { Aug., sowing of } 2 \text { seedling/ hill at } \\
20 \mathrm{~cm} \times 20 \mathrm{~cm} \text { spacing }\end{array}$ & 1.90 & 10.43 & 0.0450 & 0.0537 \\
\hline & F-test & NS & $\mathrm{S}$ & NS & NS \\
\hline & C.D. $(P=0.05)$ & - & 1.11 & - & - \\
\hline
\end{tabular}


Table.2a Effect of different transplanting dates, spacing and No. of seedlings/hill on the yield attributes and yield of hybrid rice

\begin{tabular}{|c|c|c|c|c|}
\hline & Treatments combination & $\begin{array}{c}\text { No. of } \\
\text { effective } \\
\text { tiller / hill }\end{array}$ & $\begin{array}{l}\text { Length of } \\
\text { panicle }(\mathrm{cm})\end{array}$ & $\begin{array}{l}\text { No. of grains } \\
\text { / panicle }\end{array}$ \\
\hline $\mathrm{T}_{1}$ & $\begin{array}{l}15^{\text {th }} \text { July, sowing of } 1 \text { seedling/ hill at } 20 \mathrm{~cm} \mathrm{x} \\
10 \mathrm{~cm} \text { spacing }\end{array}$ & 18.77 & 23.17 & 160.44 \\
\hline $\mathrm{T}_{2}$ & $\begin{array}{l}15^{\text {th }} \text { July, sowing of } 2 \text { seedling/ hill at } 20 \mathrm{~cm} \mathrm{x} \\
10 \mathrm{~cm} \text { spacing }\end{array}$ & 19.17 & 23.33 & 162.67 \\
\hline $\mathrm{T}_{3}$ & $\begin{array}{l}15^{\text {th }} \text { July, sowing of } 1 \text { seedling/ hill at } 20 \mathrm{~cm} \mathrm{x} \\
15 \mathrm{~cm} \text { spacing }\end{array}$ & 21.55 & 24.17 & 176.17 \\
\hline $\mathrm{T}_{4}$ & $\begin{array}{l}15^{\text {th }} \text { July, sowing of } 2 \text { seedling/ hill at } 20 \mathrm{~cm} \mathrm{x} \\
15 \mathrm{~cm} \text { spacing }\end{array}$ & 21.88 & 24.33 & 179.00 \\
\hline $\mathrm{T}_{5}$ & $\begin{array}{l}15^{\text {th }} \text { July, sowing of } 1 \text { seedling/ hill at } 20 \mathrm{~cm} \mathrm{x} \\
20 \mathrm{~cm} \text { spacing }\end{array}$ & 29.55 & 25.67 & 193.55 \\
\hline $\mathrm{T}_{6}$ & $\begin{array}{l}15^{\text {th }} \text { July, sowing of } 2 \text { seedling/ hill at } 20 \mathrm{~cm} \mathrm{x} \\
20 \mathrm{~cm} \text { spacing }\end{array}$ & 33.27 & 27.33 & 202.33 \\
\hline $\mathrm{T}_{7}$ & $\begin{array}{l}27^{\text {th }} \text { July, sowing of } 1 \text { seedling/ hill at } 20 \mathrm{~cm} \mathrm{x} \\
10 \mathrm{~cm} \text { spacing }\end{array}$ & 18.00 & 22.27 & 155.67 \\
\hline $\mathrm{T}_{8}$ & $\begin{array}{l}27^{\text {th }} \text { July, sowing of } 2 \text { seedling/ hill at } 20 \mathrm{~cm} x \\
10 \mathrm{~cm} \text { spacing }\end{array}$ & 18.33 & 23.07 & 158.00 \\
\hline $\mathrm{T}_{9}$ & $\begin{array}{l}27^{\text {th }} \text { July, sowing of } 1 \text { seedling/ hill at } 20 \mathrm{~cm} \mathrm{x} \\
15 \mathrm{~cm} \text { spacing }\end{array}$ & 20.17 & 23.88 & 170.33 \\
\hline $\mathrm{T}_{10}$ & $\begin{array}{l}27^{\text {th }} \text { July, sowing of } 2 \text { seedling/ hill at } 20 \mathrm{~cm} x \\
15 \mathrm{~cm} \text { spacing }\end{array}$ & 21.27 & 24.00 & 173.27 \\
\hline $\mathrm{T}_{11}$ & $\begin{array}{l}27^{\text {th }} \text { July, sowing of } 1 \text { seedling/ hill at } 20 \mathrm{~cm} \mathrm{x} \\
20 \mathrm{~cm} \text { spacing }\end{array}$ & 25.33 & 24.88 & 187.33 \\
\hline $\mathrm{T}_{12}$ & $\begin{array}{l}27^{\text {th }} \text { July, sowing of } 2 \text { seedling/ hill at } 20 \mathrm{~cm} \mathrm{x} \\
20 \mathrm{~cm} \text { spacing }\end{array}$ & 27.07 & 25.17 & 190.44 \\
\hline $\mathrm{T}_{13}$ & $\begin{array}{l}9^{\text {th }} \text { Aug., sowing of } 1 \text { seedling/ hill at } 20 \mathrm{~cm} \mathrm{x} \\
10 \mathrm{~cm} \text { spacing }\end{array}$ & 17.07 & 20.27 & 150.67 \\
\hline $\mathrm{T}_{14}$ & $\begin{array}{l}9^{\text {th }} \text { Aug., sowing of } 2 \text { seedling/ hill at } 20 \mathrm{~cm} \mathrm{x} \\
10 \mathrm{~cm} \text { spacing }\end{array}$ & 17.67 & 22.17 & 153.55 \\
\hline $\mathrm{T}_{15}$ & $\begin{array}{l}9^{\text {th }} \text { Aug., sowing of } 1 \text { seedling/ hill at } 20 \mathrm{~cm} \mathrm{x} \\
15 \mathrm{~cm} \text { spacing }\end{array}$ & 19.55 & 23.55 & 165.00 \\
\hline $\mathrm{T}_{16}$ & $\begin{array}{l}9^{\text {th }} \text { Aug., sowing of } 2 \text { seedling/ hill at } 20 \mathrm{~cm} \mathrm{x} \\
15 \mathrm{~cm} \text { spacing }\end{array}$ & 19.88 & 23.67 & 167.27 \\
\hline $\mathrm{T}_{17}$ & $\begin{array}{l}9^{\text {th }} \text { Aug., sowing of } 1 \text { seedling/ hill at } 20 \mathrm{~cm} \mathrm{x} \\
20 \mathrm{~cm} \text { spacing }\end{array}$ & 22.67 & 24.55 & 182.17 \\
\hline $\mathrm{T}_{18}$ & $\begin{array}{l}9^{\text {th }} \text { Aug., sowing of } 2 \text { seedling/ hill at } 20 \mathrm{~cm} \mathrm{x} \\
20 \mathrm{~cm} \text { spacing }\end{array}$ & 23.67 & 24.67 & 185.27 \\
\hline & F-test & $\mathrm{S}$ & $\mathrm{S}$ & $\mathrm{S}$ \\
\hline & C.D. $(P=0.05)$ & 0.24 & 0.26 & 1.89 \\
\hline
\end{tabular}


Table.2b Effect of different transplanting dates, spacing and No. of seedlings/hill on the yield attributes and yield of hybrid rice

\section{Treatments combinations}

\section{Grain Yield}

$\left(\mathrm{t} \mathrm{ha}^{-1}\right)$
Test weight

(g)

19.17

19.33

22.33

23.67

29.67

6.43

32.55

4.27

18.44

4.40

18.88

4.36

4.62

5.62

5.83

3.85

4.03

4.86

4.17

5.23

5.46

$\mathrm{S}$

20.44

20.77

27.55

29.33

14.67

16.27

42.38

(\%)

42.40

42.40

42.39

42.50

43.19

44.37

42.43

42.36

42.40

42.68

43.34

40.66

$20 \mathrm{~cm} \times 10 \mathrm{~cm}$ spacing

41.73

$20 \mathrm{~cm} \times 10 \mathrm{~cm}$ spacing

$\mathrm{T}_{15} \quad 9^{\text {th }}$ Aug., sowing of 1 seedling/ hill at $20 \mathrm{~cm} \times 15 \mathrm{~cm}$ spacing

$\mathrm{T}_{16} \quad 9^{\text {th }}$ Aug., sowing of 2 seedling/ hill at $20 \mathrm{~cm} \times 15 \mathrm{~cm}$ spacing

$\mathrm{T}_{17} \quad 9^{\text {th }}$ Aug., sowing of 1 seedling/ hill at $20 \mathrm{~cm} \times 20 \mathrm{~cm}$ spacing

$\mathrm{T}_{18} \quad 9^{\text {th }}$ Aug., sowing of 2 seedling/ hill at $20 \mathrm{~cm}$ x $20 \mathrm{~cm}$ spacing F-test

C.D. $(P=0.05)$

19.67

42.39

42.39

42.17

42.38

25.88

NS 
Table.3 Effect of different transplanting dates, spacing and No. of seedlings/hill on the economics of hybrid rice

\begin{tabular}{|c|c|c|c|c|c|}
\hline S.No. & Treatments combination & $\begin{array}{l}\text { Cost of } \\
\text { cultivation } \\
\left(\mathrm{ha}^{-1}\right)\end{array}$ & $\begin{array}{l}\text { Gross } \\
\text { return } \\
\left(\mathrm{ha}^{-1}\right)\end{array}$ & $\begin{array}{l}\text { Net return } \\
\left(\mathrm{ha}^{-1}\right)\end{array}$ & $\mathrm{B}: \mathrm{C}$ ratio \\
\hline $\mathrm{T}_{1}$ & $\begin{array}{l}15^{\text {th }} \text { July, sowing of } 1 \text { seedling/ } \\
\text { hill at } 20 \mathrm{~cm} \times 10 \mathrm{~cm} \text { spacing }\end{array}$ & 50754.47 & 63996.00 & 13241.53 & 1.26 \\
\hline $\mathrm{T}_{2}$ & $\begin{array}{l}15^{\text {th }} \text { July, sowing of } 2 \text { seedling/ } \\
\text { hill at } 20 \mathrm{~cm} \times 10 \mathrm{~cm} \text { spacing }\end{array}$ & 54022.60 & 66454.00 & 12431.40 & 1.23 \\
\hline $\mathrm{T}_{3}$ & $\begin{array}{l}15^{\text {th }} \text { July, sowing of } 1 \text { seedling/ } \\
\text { hill at } 20 \mathrm{~cm} \times 15 \mathrm{~cm} \text { spacing }\end{array}$ & 49618.41 & 71130.00 & 21511.59 & 1.43 \\
\hline $\mathrm{T}_{4}$ & $\begin{array}{l}15^{\text {th }} \text { July, sowing of } 2 \text { seedling/ } \\
\text { hill at } 20 \mathrm{~cm} \times 15 \mathrm{~cm} \text { spacing }\end{array}$ & 52248.47 & 74514.00 & 22265.53 & 1.42 \\
\hline $\mathrm{T}_{5}$ & $\begin{array}{l}15^{\text {th }} \text { July, sowing of } 1 \text { seedling/ } \\
\text { hill at } 20 \mathrm{~cm} \times 20 \mathrm{~cm} \text { spacing }\end{array}$ & 48637.97 & 88178.00 & 39540.03 & 1.81 \\
\hline $\mathrm{T}_{6}$ & $\begin{array}{l}15^{\text {th }} \text { July, sowing of } 2 \text { seedling/ } \\
\text { hill at } 20 \mathrm{~cm} \times 20 \mathrm{~cm} \text { spacing }\end{array}$ & 50816.45 & 93330.00 & 42513.55 & 1.83 \\
\hline $\mathrm{T}_{7}$ & $\begin{array}{l}27^{\text {th }} \text { July, sowing of } 1 \text { seedling/ } \\
\text { hill at } 20 \mathrm{~cm} \times 10 \mathrm{~cm} \text { spacing }\end{array}$ & 50754.47 & 60070.00 & 9315.53 & 1.18 \\
\hline $\mathrm{T}_{8}$ & $\begin{array}{l}27^{\text {th }} \text { July, sowing of } 2 \text { seedling/ } \\
\text { hill at } 20 \mathrm{~cm} \times 10 \mathrm{~cm} \text { spacing }\end{array}$ & 54022.60 & 62054.00 & 8031.14 & 1.14 \\
\hline $\mathrm{T}_{9}$ & $\begin{array}{l}27^{\text {th }} \text { July, sowing of } 1 \text { seedling/ } \\
\text { hill at } 20 \mathrm{~cm} \times 15 \mathrm{~cm} \text { spacing }\end{array}$ & 49618.41 & 64270.00 & 14651.59 & 1.29 \\
\hline $\mathrm{T}_{10}$ & $\begin{array}{l}27^{\text {th }} \text { July, sowing of seedling/ } \\
\text { hill at } 20 \mathrm{~cm} \times 15 \mathrm{~cm} \text { spacing }\end{array}$ & 52248.47 & 67958.00 & 15709.53 & 1.30 \\
\hline $\mathrm{T}_{11}$ & $\begin{array}{l}27^{\text {th }} \text { July, sowing of } 1 \text { seedling/ } \\
\text { hill at } 20 \mathrm{~cm} \times 20 \mathrm{~cm} \text { spacing }\end{array}$ & 48637.97 & 82492.00 & 33854.03 & 1.69 \\
\hline $\mathrm{T}_{12}$ & $\begin{array}{l}27^{\text {th }} \text { July, sowing of } 2 \text { seedling/ } \\
\text { hill at } 20 \mathrm{~cm} \times 20 \mathrm{~cm} \text { spacing }\end{array}$ & 50816.45 & 85250.00 & 34433.55 & 1.67 \\
\hline $\mathrm{T}_{13}$ & $\begin{array}{l}9^{\text {th }} \text { Aug., sowing of } 1 \text { seedling/ } \\
\text { hill at } 20 \mathrm{~cm} \times 10 \mathrm{~cm} \text { spacing }\end{array}$ & 50754.47 & 54594.00 & 3839.53 & 1.07 \\
\hline $\mathrm{T}_{14}$ & $\begin{array}{l}9^{\text {th }} \text { Aug., sowing of } 2 \text { seedling/ } \\
\text { hill at } 20 \mathrm{~cm} \times 10 \mathrm{~cm} \text { spacing }\end{array}$ & 54022.60 & 56778.00 & 2755.40 & 1.05 \\
\hline $\mathrm{T}_{15}$ & $\begin{array}{l}9^{\text {th }} \text { Aug., sowing of } 1 \text { seedling/ } \\
\text { hill at } 20 \mathrm{~cm} \times 15 \mathrm{~cm} \text { spacing }\end{array}$ & 49618.41 & 68914.00 & 19295.59 & 1.38 \\
\hline $\mathrm{T}_{16}$ & $\begin{array}{l}9^{\text {th }} \text { Aug., sowing of } 2 \text { seedling/ } \\
\text { hill at } 20 \mathrm{~cm} \times 15 \mathrm{~cm} \text { spacing }\end{array}$ & 52248.47 & 61478.00 & 9229.53 & 1.17 \\
\hline $\mathrm{T}_{17}$ & $\begin{array}{l}9^{\text {th }} \text { Aug., sowing of } 1 \text { seedling/ } \\
\text { hill at } 20 \mathrm{~cm} \times 20 \mathrm{~cm} \text { spacing }\end{array}$ & 48637.97 & 82058.00 & 33420.03 & 1.68 \\
\hline $\mathrm{T}_{18}$ & $\begin{array}{l}9^{\text {th }} \text { Aug., sowing of } 2 \text { seedling/ } \\
\text { hill at } 20 \mathrm{~cm} \times 20 \mathrm{~cm} \text { spacing }\end{array}$ & 50816.45 & 80470.00 & 29653.53 & 1.58 \\
\hline
\end{tabular}


Similar results have been also reported by Paliwal et al., (1996) and Singh et al., (2004).

\section{Test weight (g)}

The observations on response of hybrid rice on the test weight were statistically analyzed and are being presented in table 3 . A critical review of the table clearly shows that the significantly highest test weight $(32.55 \mathrm{~g})$ was recorded under treatment $\mathrm{T}_{6}(15 \mathrm{July}+20 \mathrm{x} 20$ $\mathrm{cm}+2$ Seedling hill $\left.{ }^{-1}\right)$.

Test weight, was significantly higher in $15^{\text {th }}$ July planted crop than the $30^{\text {th }}$ July and $14^{\text {th }}$ August planted crop which might be due to availability of more time for better development of plant parts which may result in the better development of yield attributing characters. Similar results have been also reported by Paliwal et al., (1996) and Singh et al., (2004).

\section{Grain yield t/ha}

The observations on response of hybrid rice on grain yield were statistically analyzed and are being presented in table 3. A critical review of the table clearly shows that the significantly highest grain yield $(6.43 \mathrm{t} / \mathrm{ha})$ was recorded under treatment $\mathrm{T}_{6}$ (15 July $+20 \times 20 \mathrm{~cm}+2$ Seedling hill ${ }^{-1}$ ).

Planting on $16^{\text {th }}$ July recorded the maximum grain yield. A fortnight delay in planting from $16^{\text {th }}$ July reduced the grain yield by 7.6 and 3.3 percent during the first and second year respectively. One day delay in planting, on an average, reduced the grain yield by $14.5 \mathrm{~kg}$ and one month delay in planting from $16^{\text {th }}$ July reduced the grain yield by 24.3 percent. Per day reduction on grain yield was $38 \mathrm{~kg} / \mathrm{ha}$ respectively. The results confirms the findings of Parihar et al., (1995), Pandey et al., (2001) and Nayak et al., (2003) and Mahajan et al., (2010).
It may be concluded that $15^{\text {th }}$ July of transplanting, 2 seedlings of rice hill ${ }^{-1}$ at $20 \mathrm{~cm} \times 20 \mathrm{~cm}$ spacing was found to be the best for obtaining highest grain yield and benefit cost ratio in hybrid rice Arise 6444 . Although these findings are based on one year experimentation, therefore further trials are required to confirm the findings.

\section{References}

Bruns, H.A, Abbas, H.K. (2006). Planting date effects on Bt and Non-Bt corn in the mid-south USA. Agron. J., 98: 100-106.

Gautam, A. K. Kumar, Dinesh, Shivay, Y.S. and Mishra B.N.(2008) (a). Influence of nitrogen levels and plant spacing on growth, productivity and quality of two inbred varieties and a hybrid of aromatic rice. Archives of agronomy and soil siience, 54 (5): 515-532.

Gautam, A. K. Kumar, Dinesh, Shivay, Y.S. and Mishra B.N.(2008) (b). Influence of nitrogen levels and plant spacing on growth, productivity and quality of two inbred varieties and a hybrid of aromatic rice. Archives of agronomy and soil siience, 54 (5): 515-532.

Kumar, M.R. Subbaiah, S.V. and Pillai, G.K. (1998). Response of rice hybrids to different dates of planting in vertisols of Andhra Pradesh, Oryza, 35 (3): 285287.

Mahajan, G. Singh, Naveen and Kaur, Rupinder (2010). Optimization of transplanting time, nitrogen requirement and plant spacing for higher yield of Basmati rice cultivars in Punjab. Environment and Ecology. 2010. 28: 2, 770-774.

Nayak, B.C. Dalei, B.B. and Choudhury, B.K. (2003) (a). Response of hybrid rice to dates of planting, spacing and seedling rate during wet season. Indian J. Agron. 48 (3): 172-174. 
Nayak, B.C. Dalei, B.B. and Choudhury, B.K. (2003) (b). Response of hybrid rice to dates of planting, spacing and seedling rate during wet season. Indian J. Agron. 48 (3): 172-174.

Paddalia, C.R. (1981). Effect of age of seedling on the growth and yield of transplanted rice. Oryza, 18: 165-167.

Paliwal, A.K Khandalker, V.S. Paraye, P.M. and Tomar, N.S. (1996) (a). Effect of transplanting time on grain yield and its attributes in dwarf scented rice. Indian J. Agric. Sci., 66(1): 48-50.

Paliwal, A.K Khandalker, V.S. Paraye, P.M. and Tomar, N.S. (1996) (b). Effect of transplanting time on grain yield and its attributes in dwarf scented rice. Indian J. Agric. Sci., 66(1): 48-50.

Parihar, S.S. Verma, V.K. Shukla, R.K. Pandey, D. and Sharma, R.B. (1995) (a). Response of transplanted rice to planting time and nitrogen schedule. Indian J. Agron., 40 (3): 402-406.

Parihar, S.S. Verma, V.K. Shukla, R.K. Pandey, D. and Sharma, R.B. (1995) (b). Response of transplanted rice to planting time and nitrogen schedule. Indian J. Agron., 40 (3): 402-406.

Pandey, N. Verma, A.K. and Tripathi, R.S. (2001) (a). Effect of planting dates and nitrogen on tillering pattern, dry matter accumulation and grain yield of hybrid rice. Indian J. Agril. Sci. 71 (5): 337338.

Pandey, N. Verma, A.K. and Tripathi, R.S. (2001) (b). Effect of planting dates and nitrogen on tillering pattern, dry matter accumulation and grain yield of hybrid rice. Indian J. Agril. Sci. 71 (5): 337338.

Sha X, Linscombe, S.D. (2005). Planting date is critical for maximizing rice yield and milling quality. Louisiana Agric., 48(3): 6-8.

Yadav, V.K. and Tripathi, H.N. (2008) (a). Effect of date of planting, plant geometry and number of seedlings on growth and yield of hybrid rice. Department of agronomy C.S.A.U.\&T Kanpur,208 002.

Yadav, V.K. and Tripathi, H.N. (2008) (b). Effect of date of planting, plant geometry and number of seedlings on growth and yield of hybrid rice. Department of agronomy C.S.A.U.\&T Kanpur,208 002.

Yadav, V.K. and Tripathi, H.N. (2008) (c). Effect of date of planting, plant geometry and number of seedlings on growth and yyield of hybrid rice. Department of agronomy C.S.A.U.\&T Kanpur,208 002.

\section{How to cite this article:}

Shivam Dubey, Rajesh Singh, A.C. Singh and Vishwakarma, S.P. 2017. Response of Hybrid Rice (Oryza sativa L.) to Different Dates of Transplanting, Spacing and Number of Seedlings/Hill. Int.J.Curr.Microbiol.App.Sci. 6(6): 1936-1945. doi: https://doi.org/10.20546/ijcmas.2017.606.226 